\title{
Effects of platelet-derived growth factor on chondrocyte proliferation, migration and apoptosis via regulation of GIT1 expression
}

\author{
GUANG-ZONG ZHAO $^{1 *}$, LONG-QIANG ZHANG ${ }^{1 *}$, YAO LIU $^{2}$, JUN FANG $^{1}$, \\ HUA-ZHUANG LI ${ }^{1}$, KE-HAI GAO ${ }^{1}$ and YUN-ZHEN CHEN $^{3}$ \\ Departments of ${ }^{1}$ Orthopedics and ${ }^{2}$ Gynecology, Yidu Central Hospital of Weifang, Weifang, \\ Shandong 262500; ${ }^{3}$ Department of Orthopedics, Qilu Hospital of Shandong University, \\ Jinan, Shandong 250012, P.R. China
}

Received April 7, 2015; Accepted April 19, 2016

DOI: $10.3892 / \mathrm{mmr} .2016 .5291$

\begin{abstract}
The formation of fibrocartilage, cartilaginous and bony calluses is vital for bone healing following a fracture. Fibroblasts, chondrocytes and osteoblasts are critical functional cells that are involved in these three processes, respectively. Platelet-derived growth factor (PDGF), a growth factor that is released from platelet particles and appears during the early stages at the site of fractures, is essential in bone healing via regulation of cell proliferation and differentiation. However, the effects of PDGF on the chondrocytes remain unclear. In the present study, PDGF promoted phosphorylation of Src and upregulated the expression level of G-protein-coupled receptor kinase interacting protein-1 (GIT1) according to the results of the cell culture of chondrocytes in vitro and western blotting. However, the effect of PDGF on the upregulation of GIT1 expression was mostly inhibited by the Src inhibitor, PP2. After knocking down GIT1 expression using siRNA, the phosphorylation of Src continued to be induced by PDGF, although the expression of GIT1 was inhibited. Furthermore, the results indicated that PDGF promoted chondrocyte proliferation and migration, however, the effect on cell apoptosis induction was suppressed after adding the Src inhibitor, PP2. Additionally, when knocking down GIT1 using siRNA, the expression level of GIT1 decreased, which is similar to the effect of the Src inhibitor, PP2. The current study demonstrates that PDGF may initially activate the phosphorylation of Src, and subsequently
\end{abstract}

Correspondence to: Dr Yun-Zhen Chen, Department of Orthopedics, Qilu Hospital of Shandong University, 107 Wenhua West Road, Jinan, Shandong 250012, P.R. China

E-mail: yzchen6501@hotmail.com

*Contributed equally

Key words: platelet-derived growth factor, G-protein-coupled receptor kinase interacting protein-1, chondrocyte, proliferation induce GIT1 expression to promote chondrocyte proliferation and migration, but suppress cell apoptosis.

\section{Introduction}

Bone healing is characterized by different processes consisting of the recruitment, migration, proliferation and differentiation of repair cells, as well as autocrine and paracrine mechanisms. Platelet-derived growth factor (PDGF) is a cell growth factor that is released from platelet particles and presents near to damaged tissues during the early stage of fracture healing. PDGF, which a $30-\mathrm{kDa}$ dimer of $\mathrm{A}$ and $\mathrm{B}$ polypeptide chains linked by disulphide bonds, is critical in regulating osteoblast replication and bone collagen degradation, controlling the proliferation of repair cells, and inducing cartilage and bone formation (1). As a multiple mitogen, PDGF is released by platelets and facilitates blood clotting via the adhesion between platelets and blood vessels. Previous studies demonstrated that PDGF was a stimulator for bone fracture healing and was responsible for bone metabolism processes, including cell proliferation, migration and apoptosis $(2,3)$. Furthermore, PDGF has three isoforms comprising of PDGF-AA, -BB and $-\mathrm{AB}$ for bone metabolism and bone remodeling (3). Nash et al (4) demonstrated that the bone marrow cavity volume and bone mineral density were markedly increased after injecting recombinant human PDGF-BB into rabbits with tibial fractures, indicating that PDGF participates in the stimulation of fracture healing. In addition, cartilage cells cultured with PDGF-BB and transforming growth factor $\beta 1$ (TGF- $\beta 1$ ) exhibited a significantly higher proliferation rate in the study by Brandl et al (3). Furthermore, the findings of Lee et al (5) and Schmidt et al (6) support the hypothesis that PDGF promotes the proliferation of cultured cartilage cells and repairs cartilage tissue.

G-protein-coupled receptor kinase interacting protein-1 (GIT1) has been identified in various mammals and birds (7). A yeast two-hybrid screen was originally used to identify GIT1, which binds to $G$ protein-coupled receptor kinase (GRK2) for activation and membrane recruitment. GIT1 was identified to be essential in focal adhesions, and cell growth and migration, 
as it was located in the cytoplasmic membrane and composite structures, such as inclusion bodies (8-11). GIT1, a scaffold protein, has been demonstrated to act as a GTPase-activating protein for the ADP-ribosylation factor family of proteins in previous studies (8-11). The results of recent studies have shown that GIT1 is important in receptor endocytosis and cell motility $(11,12)$. Additionally, GIT1 is responsible for phosphorylation of phospholipase $\mathrm{C} \gamma(\mathrm{PLC} \gamma 1)$ and activation of extracellular signal-regulated kinase (ERK)1/2 signaling induced by angiotensin II and epidermal growth factor (12). Previously, it was found that GIT1 regulated cytoskeletal dynamics for cell growth and migration. For example, cytoskeletal proteins, such as Paxillin, focal adhesion kinase (FAK) and Src all participate in the promotion of cell migration (13). Src, characterized by protein tyrosine kinase activity, is one of many signal transduction pathways and upstream core regulators, that is important in cell proliferation, migration and differentiation in addition to other mechanisms (13). It was identified that Src, acting as a regulating kinase, is located at ERK1/2 upstream in the signal transduction pathway of myocardial cells and nerve cells (14).

It is hypothesized that PDGF stimulates DNA replication and protein synthesis of osteoblasts and chondrocytes, and promotes osteoblast arrested in the $G_{0} / G_{1}$ phase to enter the $\mathrm{S}$ phase, inducing the cell division and proliferation of osteoblasts (1). Ren et al (15) revealed that phosphorylation of GIT1 tyrosine 321 is critical in order to associate with FAK and activate FAK in focal adhesions to promote PDGF-induced osteoblastic cell migration. In addition, Rui et al (9) indicated that the phosphorylation of GIT1Y321 is critical for ERK1/2- and PDGF-dependent vascular endothelial growth factor (VEGF) secretion from osteoblasts for the purpose of angiogenesis and bone healing stimulation. Thus, PDGF is important in the activation of GIT1 during bone repair. PDGF may promote osteoblast migration through regulating cell division control protein 42 homolog/Rac and Src activity in the phosphatidylinositol kinase signaling pathway (16). The activation of Src and its associated family members requires phosphorylation of tyrosine residues located in the membrane proximal region of the PDGF receptor (PDGFR). Src may undergo conformational reorganization of the Src kinase domain through the connection between its $\mathrm{SH} 2$ domain and the phosphorylated PDGFR in an attempt to activate Src (17). Furthermore, Src-induced formation of the calpain/FAK/ERK1/2 complex and ERK1/2 activation are required for calpain-mediated proteolysis of FAK (17). Therefore, PDGF may mediate GIT1 via the regulation of Src.

With regard to GIT1 and PDGF in bone cells, there has been less research into the effects of GIT1 and PDGF on cell proliferation and cell apoptosis in chondrocytes compared with cell migration in osteoblasts. PDGF may activate specific tyrosine kinases containing PDGF- $\alpha$ and $-\beta$ when the corresponding receptors are present on the cell membrane, contributing to increased mitogenic signals in the signal transduction pathway and leading to DNA synthesis, cell division and proliferation induction (18). Furthermore, PDGF has been demonstrated to be critical in the ERK1/2 signaling pathway. Thus, PDGF may activate the cytokine-mediated ERK1/2 signaling pathway to regulate the typical early signaling pathways associated with cell proliferation, differentiation and apoptosis.

\section{Materials and methods}

Cell culture. Animal care and use followed the ethical guidelines of the Chinese Council on Animal Care and were reviewed and approved by the Institutional Animal Care and Use Committee of Qilu Hospital of Shandong University (Jinan, China). Three one-week-old Sprague-Dawley neonatal rats were purchased from the Institute of Laboratory Animal Sciences, Chinese Academy of Medical Sciences and Peking Union Medical College (Beijing, China). The weight of the rats was 300-350 g. The rats were sacrificed by an intraperitoneal injection of euthatal solution $(150 \mathrm{mg} / \mathrm{kg}$; Sigma-Aldrich, St. Louis, MO, USA) and then dissected aseptically to obtain all the limb joints. The fibrous tissue was removed from the articular cartilage surface of the limb joints and the transparent central portion of cartilage was sliced into small sections (size, $\sim 1 \mathrm{~mm}^{3}$ ). The small tissue sections were digested using $0.25 \%$ trypsin (Gibco; Thermo Fisher Scientific, Inc., Waltham, MA, USA) at $37^{\circ} \mathrm{C}$ for $30 \mathrm{~min}$. The mixture was centrifuged at $1,000 \mathrm{x} \mathrm{g}$ for $5 \mathrm{~min}$ to obtain cell pellets. The pellets were combined with $0.2 \%$ II collagenase (Sigma-Aldrich) and incubated at $37^{\circ} \mathrm{C}$ for $4 \mathrm{~h}$ for digestion. A 200-mesh filter (Anping County Sheng Silk Factory, Tainan, China) was used and a further centrifugation at $1,000 \mathrm{x}$ g for $5 \mathrm{~min}$ was conducted to collect the cells. Invitrogen Dulbecco's modified Eagle's medium (DMEM; Thermo Fisher Scientific, Inc.) containing Gibco $10 \%$ fetal bovine serum (FBS; Thermo Fisher Scientific, Inc.), $100 \mathrm{U} / \mathrm{ml}$ penicillin (Gibco; Thermo Fisher Scientific,Inc.) and $50 \mathrm{U} / \mathrm{ml}$ streptomycin (Gibco; Thermo Fisher Scientific, Inc.) was used for maintaining the cells, which were incubated at $37^{\circ} \mathrm{C}$ in a humidified incubator with a $5 \% \mathrm{CO}_{2}$ atmosphere. After repeated isolation and culture, the morphology of the purified cells was observed under an inverted phase contrast microscope (CKX41; Olympus Corporation, Tokyo, Japan).

Transfection of siRNA. The siRNA transfection was conducted using Invitrogen Lipofectamine 2000 (Thermo Fisher Scientific, Inc.) according to the manufacturer's instructions. Briefly, the cells was seeded into 6 -well plates $\left(1 \times 10^{5}\right.$ cells $\left./ \mathrm{ml}\right)$ and incubated for $24 \mathrm{~h}$ at $37^{\circ} \mathrm{C}$. The primers, designed as previously described (9) and synthesized by Ambion (Thermo Fisher Scientific, Inc.) were as follows: Sense 5'-AAGCTGCCAAGA AGAAGCTAC-3' for GIT1 siRNA and sense 5'-AATTCT CCGACACGTGTCACT-3' for the negative control siRNA. The GIT1 siRNA (100 nM) was prepared and transfected for $24 \mathrm{~h}$ as previously described (10). The Src inhibitor, PP2 (10 $\mu \mathrm{M}$; CST Biological Reagents Co., Ltd., Shanghai, China) dissolved in $100 \%$ dimethyl sulfoxide (DMSO; Enzo Life Sciences, Inc., Farmingdale, NY, USA) was used to pretreat the cells for $1 \mathrm{~h}$. The same volume of $0.1 \%$ DMSO served as a control. PDGF (10 ng/ml; Sigma-Aldrich) was added for $24 \mathrm{~h}$ to stimulate the cells. After $72 \mathrm{~h}$, the transfected cells were analyzed by quantitative polymerase chain reaction (qPCR) and western blot analysis.

Fluorescence $q P C R$. The cells were collected and total RNA was extracted using $1 \mathrm{ml}$ Trizol (Invitrogen; Thermo Fisher Scientific, Inc.). Total RNA (1 $\mu \mathrm{g})$ was used for first-strand cDNA synthesis with RevertAid First Strand cDNA Synthesis Kit (Thermo Fisher Scientific, Inc., Pittsburgh, PA, USA). 
First-strand cDNA was synthesized using $1 \mu \mathrm{l}$ of random hexamers and adjusted to a volume of $12 \mu \mathrm{l}$ using DEPC-treated water. The mix was incubated at $65^{\circ} \mathrm{C}$ for $5 \mathrm{~min}$. The following components were added in to the reaction: $4 \mu 15 \mathrm{X}$ Reaction buffer; $1 \mu \mathrm{l}$ Ribolock RNase inhibitor (20 U/ $\mu \mathrm{l}), 2 \mu \mathrm{l} \mathrm{dNTP}$ (10 mM); and $1 \mu \mathrm{l}$ RevertAid M-MuLV RT (200 U/ $\mu \mathrm{l})$. The reactions were incubated at 25,42 and $70^{\circ} \mathrm{C}$ for 5,60 and $5 \mathrm{~min}$, respectively. Then, the cDNA was used to measure of mRNA levels by qPCR using the SYBR-Green dye (Toyobo Co., Ltd., Osaka, Japan) method with $100 \mathrm{ng}$ cDNA in a $20-\mu 1$ system. The primer sequences were as follows: Forward, 5'-ATGGATGTG TATGACGAAGTG-3' and reverse, 5'-GGTTGACTGGCA GGAAGG-3' for GIT1; forward, 5'-GTCCACCTTCCAGCA GATG-3' and reverse, 5'-CTCAGTAACAGTCCGCCTAG-3' for $\beta$-actin, which was used as a reference gene. The reaction conditions were as follows: $95^{\circ} \mathrm{C}$ for $5 \mathrm{~min}$; followed by 40 cycles of $95^{\circ} \mathrm{C}$ for $30 \mathrm{sec}, 55^{\circ} \mathrm{C}$ for $30 \mathrm{sec}$ and $72^{\circ} \mathrm{C}$ for $30 \mathrm{sec}$. qPCR was performed using the Applied Biosystems 7500 Real-Time PCR system (Thermo Fisher Scientific, Inc.) All reactions were run in triplicate and performed 3 times. Quantitative analysis was performed by comparison of the $2^{-\Delta \Delta \mathrm{Cq}}$ values (19).

Western blot analysis. Total cellular proteins were extracted from the cells using lysis buffer (Beyotime Institute of Biotechnology, Haimen, China). The solution containing cells and lysis buffer was agitated at $4^{\circ} \mathrm{C}$ for 20 min for mixing. The solution was centrifuged at $10,000 \mathrm{x}$ g at $4^{\circ} \mathrm{C}$ for $10 \mathrm{~min}$ to obtain the supernatant. The protein concentration in the cell lysates was determined using a Bradford protein assay (Pierce Biotechnology, Inc., Rockford, IL, USA). SDS-PAGE containing $10 \%$ tricine gels and $20 \mu \mathrm{g}$ of protein was loaded in each lane. The proteins were separated by electrophoresis $(40 \mathrm{~V} ; 5 \mathrm{~h})$ and transferred onto nitrocellulose membranes (Pierce Biotechnology, Inc.). The membranes were incubated in a blocking buffer consisting of 5\% non-fat milk (Pierce Biotechnology, Inc.) and Tris-buffered saline with Tween-20 [TBST; Sunshine Biotechnology (Nanjing) Co., Ltd., Nanjing, China] for $1 \mathrm{~h}$. The membranes were incubated with polyclonal rabbit anti-GIT1 (1:600; cat. no. 2919; Cell Signaling Technology Inc., Danvers, MA, USA), polyclonal rabbit anti-Src antibody (1:800; cat. no. 2108; CST), polyclonal rabbit anti-p-Src (1:800; cat. no. 2105; Cell Signaling Technology Inc.) and monoclonal rabbit anti- $\beta$-actin $(1: 1,000$; cat. no. 4970; Cell Signaling Technology Inc.) antibodies in $5 \%$ non-fat milk overnight at $4^{\circ} \mathrm{C}$. The membranes were washed with TBST every $10 \mathrm{~min}$, for $30 \mathrm{~min}$. Subsequently, goat anti-rabbit horseradish peroxidase-conjugated secondary antibody (cat. no. 7074; Cell Signaling Technology, Inc.) at 1:7,000-8,000 dilution was incubated with the membranes for $1 \mathrm{~h}$ at room temperature. After washing with TBST every $10 \mathrm{~min}$ for $30 \mathrm{~min}$, the protein bands were detected using ECL Western Blotting Substrate (Pierce Biotechnology, Inc.). The western blots were scanned using a gel imaging system (UVP, LLC, Upland, CA, USA) and the gray values of the bands were measured using Gel-Pro Analyzer software (version 6.3; Media Cybernetics, Inc., Rockville, MD, USA).

5-bromo-2'-deoxyuridine (BrdU) assay of cell proliferation. To examine the roles of PDGF, Src, GIT1 and the apoptotic pathway in cell proliferation, a BrdU assay was conducted to investigate the effects of different inhibitors on cell proliferation. Briefly, the cells were seeded into 6-well plates $\left(1 \times 10^{5}\right.$ cells $\left./ \mathrm{ml}\right)$, incubated for $24 \mathrm{~h}$ at $37^{\circ} \mathrm{C}$ and siRNA transfection was conducted using Lipofectamine 2000. After pretreating the cells with $30 \mu \mathrm{M}$ PP2, or $30 \mu \mathrm{M} 0.1 \%$ DMSO for $1 \mathrm{~h}, 10 \mathrm{ng} / \mathrm{ml}$ PDGF was added to stimulate phosphorylation for $24 \mathrm{~h}$. Cell proliferation was quantified using a BrdU colorimetric immunoassay kit (Cell Proliferation ELISA kit; Roche Diagnostics GmbH, Mannheim, Germany) according to the manufacturer's protocol. Cell proliferation was expressed as the mean percentage of the control values (set at 100\%).

Terminal deoxynucleotidyl transferase dUTP nick end labeling (TUNEL)-DAPI co-staining assay. A TUNEL-DAPI co-staining assay was performed to analyze the apoptotic cells using an In Situ cell Death Detection kit (Roche Diagnostics $\mathrm{GmbH}$ ) according to the manufacturer's instructions. Briefly, $50 \mathrm{ml} \mathrm{4 \%}$ formaldehyde (Sigma-Aldrich) was used for fixation at $4^{\circ} \mathrm{C}$ for $25 \mathrm{~min}$, then the cells were washed twice with phosphate-buffered saline (PBS). Subsequently, $50 \mathrm{ml}$ $0.2 \%$ Triton X-100 (Sigma-Aldrich) was then added to cells for a 5-min incubation at room temperature. The cells were subsequently mixed with $100 \mu$ l equilibration buffer (Roche Diagnostics $\mathrm{GmbH}$ ) at room temperature for $10 \mathrm{~min}$. The cells were washed with $2 \mathrm{ml}$ saline sodium citrate (Beijing Kangpuhuiwei Technology Co., Ltd., Beijing, China) for 15 min following incubation with $50 \mu 1$ TUNEL reaction mixture (Roche Diagnostics $\mathrm{GmbH}$ ) containing $5 \mu$ l nucleotide mixture (Roche Diagnostics $\mathrm{GmbH}$ ) and $1 \mu \mathrm{l}$ terminal deoxynucleotidyl transferase (Roche Diagnostics $\mathrm{GmbH}$ ) for $60 \mathrm{~min}$ at $37^{\circ} \mathrm{C}$. Furthermore, the cells were incubated with $50 \mathrm{ml}$ $0.3 \% \mathrm{H}_{2} \mathrm{O}_{2}$ (Sigma-Aldrich) for $10 \mathrm{~min}$ and $100 \mu \mathrm{l}$ streptavidin working solution (Roche Diagnostics $\mathrm{GmbH}$ ) for $30 \mathrm{~min}$ at room temperature. The cells were incubated with $0.5 \mu \mathrm{g} / \mathrm{ml}$ DAPI in a humidified chamber in the dark for $5 \mathrm{~min}$ at room temperature. A fluorescence microscope (Nikon Eclipse 80i; Nikon Corporation, Tokyo, Japan) was used to examine and obtain images of the stained cells.

Examination of migration ability by Transwell migration assay. The Transwell migration assay is used to examine the migratory response of cells to angiogenic inducers or inhibitors. Determination of cell migration rates was conducted in a Transwell chamber (BD Biosciences, San Jose, CA, USA). DMEM $(0.6 \mathrm{ml})$ with $0.5 \%$ FBS was added to the lower chamber. The cells were diluted to $2 \times 10^{6}$ cells $/ \mathrm{ml}$ in FBS-free DMEM medium following trypsinization. Cells (100 $\mu \mathrm{l})$ were added to the upper chamber of filters precoated with Matrigel and incubated in a $5 \% \mathrm{CO}_{2}$ atmosphere at $37^{\circ} \mathrm{C}$ for $6 \mathrm{~h}$. After removing the medium, $4 \%$ glutaraldehyde (Sigma-Aldrich) was used to fix the cells on the lower side of the insert filter for $15 \mathrm{~min}$. Subsequently, a cotton swab was used to remove the remaining cells on the upper side of the filter membrane. The cells on the lower side of the insert filter were stained with $0.1 \%$ crystal violet (Sigma-Aldrich) for $10 \mathrm{~min}$. Finally, the number of cells on the lower side of the filter was counted under a microscope after washing with PBS. The cells that had migrated through the membrane were stained and counted, therefore, the migration of chondrocytes was expressed by the relative number of migrated cells. 
A

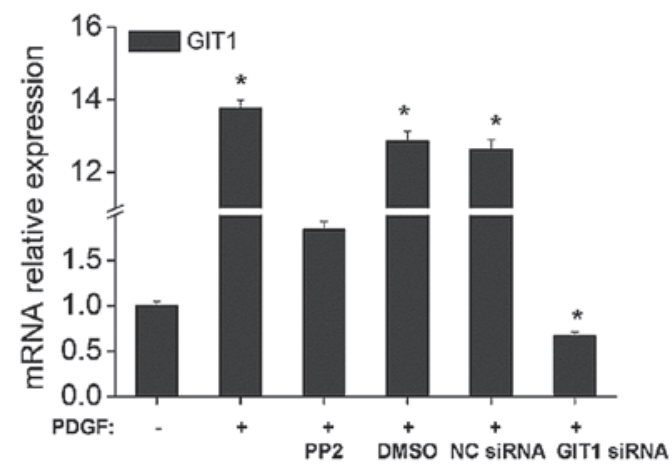

B

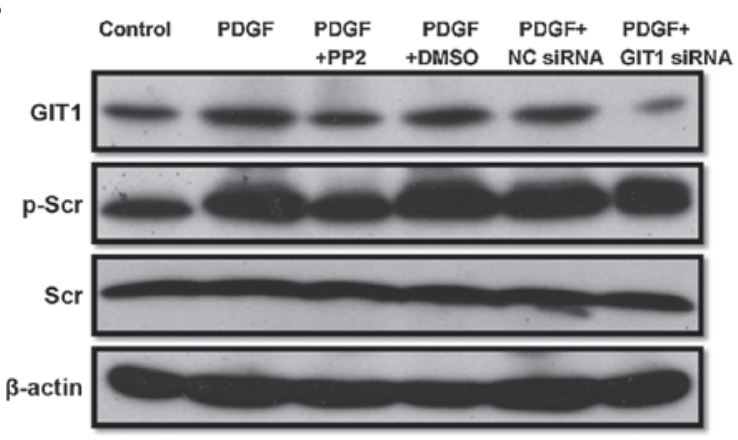

C

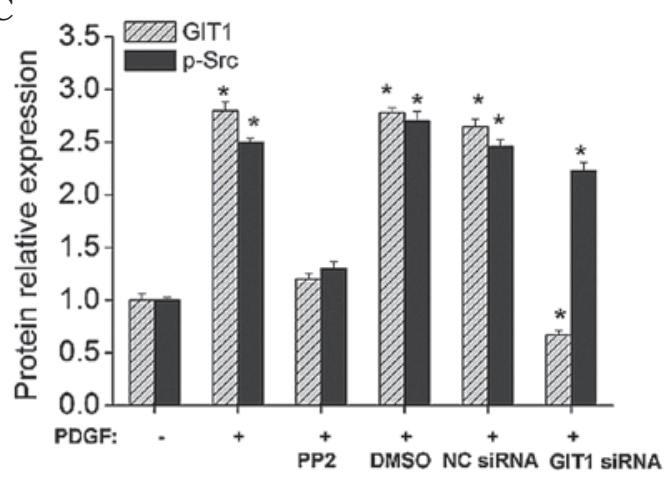

Figure 1. PDGF induced Src phosphorylation via upregulation of GIT1 expression. Cells were incubated for $24 \mathrm{~h}$ and GIT1 siRNA transfection was conducted using Lipofectamine 2000. PP2 $(30 \mu \mathrm{M})$ or $30 \mu \mathrm{M}$ DMSO was added for $1 \mathrm{~h}$ then $10 \mathrm{ng} / \mathrm{ml}$ PDGF was added for $24 \mathrm{~h}$. The mRNA and protein expression levels were examined by (A) fluorescence quantitative polymerase chain reaction and (B and C) western blotting. Experiments were performed at least in triplicate and the results were expressed as means \pm standard deviation. "P $<0.05$ vs. the control. PDGF, platelet-derived growth factor; DMSO, dimethyl sulfoxide; NC, negative control; GIT1, G-protein-coupled receptor kinase interacting protein-1; p, phosphorylated.

Statistical analysis. The experiments were performed in triplicate, at minimum, and results were expressed as means \pm standard deviation. SPSS statistical package 13.0 (SPSS, Inc., Chicago, IL, USA) was used for statistical analysis. The difference between two groups was analyzed by two-tailed Student's $t$-test and differences between three or more groups were analyzed by one-way analysis of variance and multiple comparisons. $\mathrm{P}<0.05$ was considered to indicate a statistically significant difference.

\section{Results}

PDGF induced Src phosphorylation via upregulation of GITI expression. In the present study, the roles of PDGF, GIT1 and Src were analyzed by siRNA silencing techniques and western blot analysis (Fig. 1). The western blot analysis indicated that PDGF promotes Src phosphorylation, while upregulating GIT1 expression. The results revealed that PDGF increased the expression level of GIT1 2.8-fold, thereby promoting a 2.5-fold increase in Src phosphorylation (Fig. 1B). Although PDGF was combined with cartilage cells for stimulation, the GIT1 expression level reduced to $60 \%$ of the control when GIT1 was knocked down by siRNA. However, the effects of PDGF on GIT1 overexpression were suppressed after adding the Src inhibitor, PP2. As shown in Fig. 1, the effect of PDGF on promotion of Src phosphorylation was not statistically significant, however, the expression of GIT1 was inhibited by knock down of GIT1 by siRNA. Therefore, the results demonstrate that PDGF initially activates Src phosphorylation, which in turn increases the level of GIT1 expression, suggesting that Src is upstream of GIT1.

PDGF promotes cell proliferation via regulation of GIT1 and Src. In order to investigate the biological functional roles of Src, GIT1 and PDGF in cartilage cells, the effects of their siRNA and specific inhibitors on the PDGF-activated cell proliferation were further investigated in the present study. A BrdU assay was used to analyze the cell proliferation of chondrocytes. The results demonstrated that PDGF stimulated chondrocyte proliferation by regulating GIT1 and Src (Fig. 2). According to Fig. 2, the cell proliferation of chondrocytes was significantly suppressed by the Src inhibitor, PP2. Indicating that Src is key in chondrocyte proliferation. Since Src is an upstream core regulator in various signal transduction pathways, the addition of Src inhibitor, PP2, decreased the cell proliferation ability to a greater extent than cells treated with GIT1 siRNA. However, although GIT1 expression was downregulated, cell proliferation was still greater than that of the control group. Therefore, there may be certain additional signaling pathways that involve the induction of chondrocyte proliferation by PDGF. Furthermore, the chondrocyte protein levels were greater than those of the negative control in the experiments (Fig. 1B and C), which may indicate that PDGF affects chondrocyte proliferation by mediating other proteins whilst inhibiting Src or GIT1.

PDGF affects cell apoptosis via GIT1 and Src regulation. Apoptosis is an important process in programmed cell death 
that occurs in multicellular organisms. In an attempt to examine DNA fragmentation resulting from apoptotic signaling cascades, the TUNEL-DAPI assay was used to conduct further analyses. The TUNEL assay was used to investigate the role of GIT1 in chondrocyte apoptosis depending on the presence of identifiable nicks in the DNA. As shown in Fig. 3, chondrocytes demonstrated DNA fragmentation, which was evidenced by apoptotic cell death. The results showed that the presence of PDGF markedly decreased the number of apoptotic cells. Furthermore, siRNA silencing of GIT1 resulted in the reversal of the protective effects of PDGF. Therefore, these results demonstrated that GIT1 is key in PDGF-induced chondrocyte survival.

PDGF promotes chondrocyte migration. The cell migration ability of cartilage cells induced by PDGF and GIT1 was detected by Transwell assay. The results revealed that PDGF induced chondrocyte migration ability (Fig. 4). When GIT1 was knocked down using siRNA, the effect of PDGF on the migration ability of cartilage cells was significantly suppressed (Fig. 4). This finding indicated that GIT1 is critical in chondrocyte migration.

\section{Discussion}

Local growth factors in bones and cytokines are critical in the processes of fibrocartilage, cartilaginous and bony callus formation for fracture healing following bone tissue damage (1-4). Previous research has demonstrated that various growth factors promote bone healing with synergistic effects $(5,6)$. PDGF, a growth factor (associated with healing damaged tissue) is released following bone fracture and is considered to be essential growth factor for processes, including osteogenesis and bone remodeling (1-4). Previous studies demonstrated that PDGF promotes bone cell differentiation, cell proliferation and regulation of bone cells, and participates in bone formation (20). PDGF is essential in promotion of bone formation and bone remodeling by interacting with a variety of cells during fracture healing. Furthermore, it has been demonstrated that PDGF collaborates with other factors, such as NGF, to promote fracture healing (21). The aim of the present study was to examine the effects of PDGF on chondrocyte proliferation and apoptosis via regulation of GIT1 and Src.

Differentiation, proliferation, secretory function and apoptosis of chondrocytes have been regarded as critical steps for the development, growth and remodeling of bones (22). PDGF, as well as PDGF mRNA, are apparent at the early stage of callus formation in cartilage, whereas the expression of PDGF becomes weak or disappears in the latter hypertrophy of mature chondrocytes (23). Therefore, it is essential to investigate the role of PDGF and its underlying mechanisms in cartilage cells. In the present study, the results indicated that PDGF promotes chondrocyte proliferation and migration, and suppresses apoptosis. Brandl et al (3) demonstrated that there was a markedly higher proliferation rate of chondrocytes cultured with PDGF-BB and TGF- $\beta 1$ than that of control cells. Schmidt et al (6) found that PDGF promoted chondrocyte proliferation and proteoglycan synthesis. Furthermore, the role of ectopic cartilage formation was promoted, endochondral

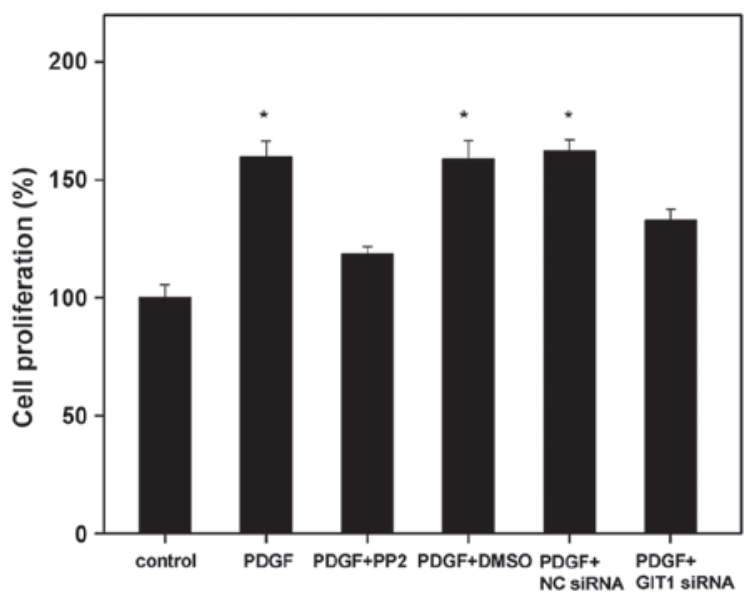

Figure 2. PDGF promoted cell proliferation via regulation of GIT1 and Src. The cells were seeded into 6-well plates and incubated for $24 \mathrm{~h}$ and transfection with GIT1 siRNA was performed using Lipofectamine 2000. After pretreating the cells with $30 \mu \mathrm{M}$ PP2 or $30 \mu \mathrm{M} 0.1 \%$ DMSO for $1 \mathrm{~h}$, $10 \mathrm{ng} / \mathrm{ml}$ PDGF was added for $24 \mathrm{~h}$. Cell proliferation was examined by 5-bromo-2'-deoxyuridine assay. " $\mathrm{P}<0.05$ vs. control. PDGF, platelet-derived growth factor; DMSO, dimethyl sulfoxide; NC, negative control; GIT1, $\mathrm{G}$-protein-coupled receptor kinase interacting protein-1.

bone formation was inhibited and chondrocyte phenotype stability was maintained without mechanical stimulation by PDGF in the experiments (6). In addition, it has been reported that PDGF is able to stimulate cultured chondrocytes and restore cartilage tissue proliferation (3-6).

A potential mechanism underlying the effects of PDGF on the proliferation, migration and apoptosis of cartilage cells is that PDGF mediates the induction of Src phosphorylation by upregulating the expression GIT1. Western blotting demonstrated that PDGF promoted Src phosphorylation, while upregulating GIT1 expression. Furthermore, the effect of PDGF on GIT1 overexpression was significantly inhibited by the presence of Src inhibitor, PP2. However, knock down of GIT1 expression using siRNA did not influence the effect of PDGF on Src phosphorylation induction. These results demonstrated that PDGF activated the phosphorylation of Src first, and subsequently increased GIT1 expression, indicating that Src is upstream of GIT1. Additionally, after using the Src inhibitor, PP2, the effect of PDGF on induction of chondrocyte proliferation was markedly decreased. Additionally, knocking down GIT1 using siRNA showed a similar result that was comparable to that of PP2. Src, one of the most important members of the Src family, is characterized by tyrosine kinase activity. The phosphorylation of $\mathrm{Src}^{-T y r}{ }^{418}$ in the Src protein kinase activation loop triggers kinase activation; activated Src results in the transfer of a phosphate group to the target molecules. As a result, the target molecule phosphorylates and transmits the signal to the nucleus, which eventually promotes mechanisms, including cell proliferation, migration and differentiation $(23,24)$. The findings of Ren et al (15) indicated that periodic mechanical stress activated the Src-PLC $\gamma 1$-mitogen-activated protein kinase kinase (MEK)1/2-ERK1/2 signaling pathway to induce chondrocyte proliferation and matrix synthesis. In addition, the study of Ren et al (25) demonstrated that the Src-PLC $\gamma 1-$ MEK1/2 signaling pathway involved chondrocyte area expansion and migration using periodic mechanical stress. 

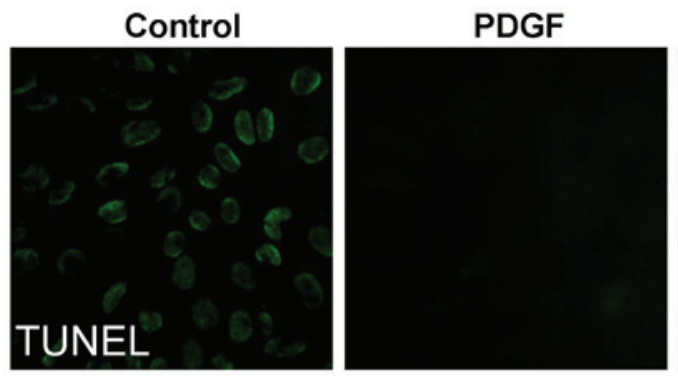

PDGF+ GIT1 siRNA
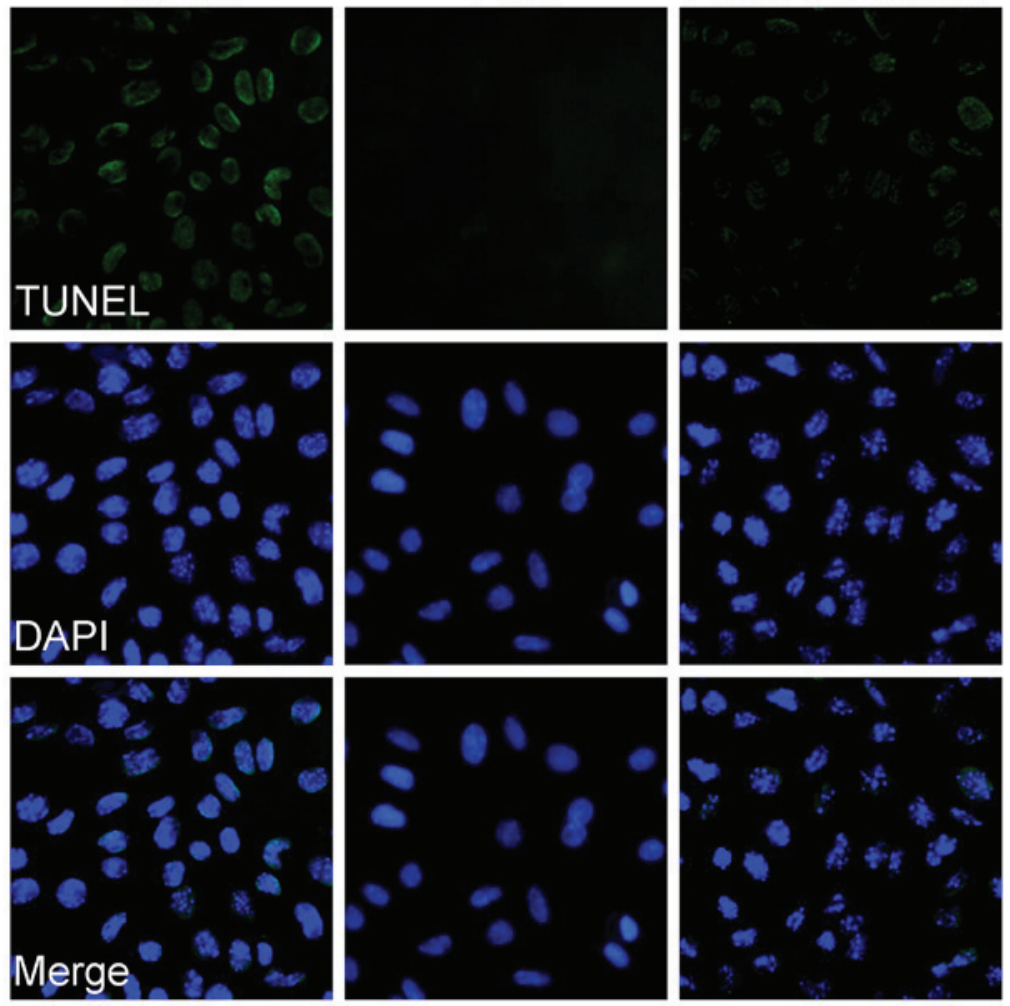

Figure 3. PDGF effects cell apoptosis via regulation of GIT1 and Src. The cells were seeded into 6-well plates and incubated for $24 \mathrm{~h}$ and GIT1 siRNA transfection was conducted using Lipofectamine 2000. Following pretreatment of the cells with various specific inhibitors, $10 \mathrm{ng} / \mathrm{ml}$ PDGF was added for $24 \mathrm{~h}$. Cell apoptosis was determined using a TUNEL-DAPI co-staining assay with apoptotic cells stained green and the nuclei stained blue. Magnification, x200. PDGF, platelet-derived growth factor; DMSO, dimethyl sulfoxide; GIT1, G-protein-coupled receptor kinase interacting protein-1; TUNEL, terminal deoxynucleotidyl transferase dUTP nick end labeling.
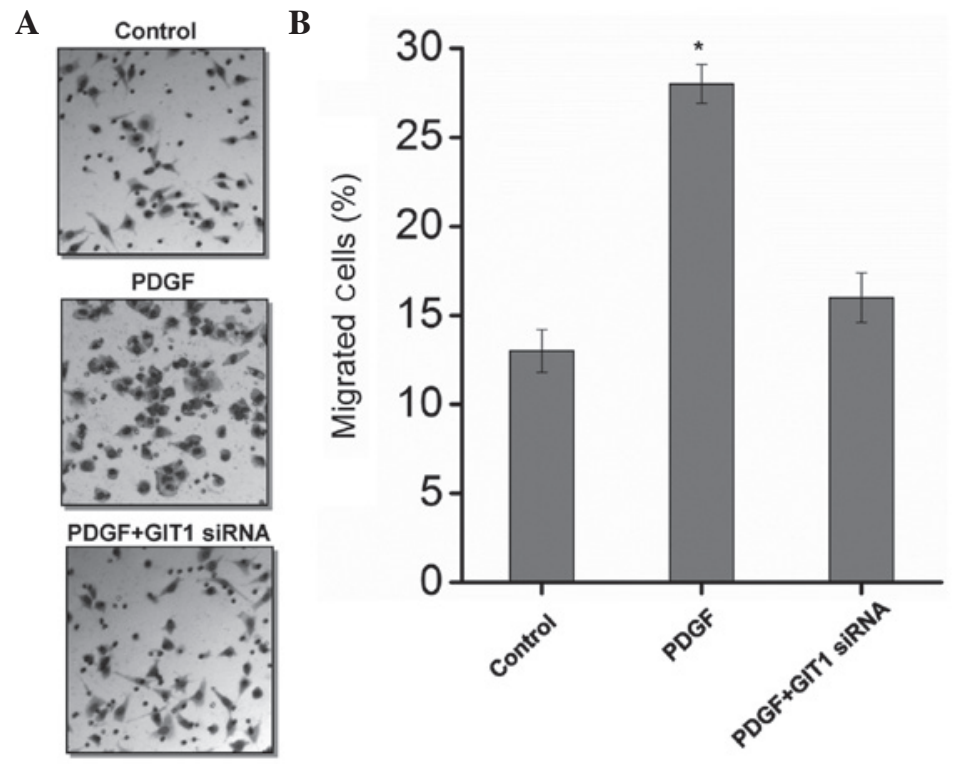

Figure 4. PDGF promotes chondrocyte migration. (A) Cell migration ability was detected by Transwell assay. The cells transfected with GIT1 siRNA were seeded onto Matrigel-coated filters of Transwell inserts and incubated for $6 \mathrm{~h}$. Subsequently, $10 \mathrm{ng} / \mathrm{ml}$ PDGF was added to 2 groups for $6 \mathrm{~h}$. The cells that migrated to the underside of the filters were counted. (B) Quantitative analysis of the migration effects of GIT1 siRNA. Each bar represents the mean \pm standard deviation from 3 samples. "P $<0.05$ vs. control. PDGF, platelet derived growth factor; GIT1, G-protein-coupled receptor kinase interacting protein-1; siRNA, mall interfering RNA.

GIT1, present in mammals and birds, is a multidomain scaffold protein that participates in the internalization and membrane transportation of $\mathrm{G}$ protein-coupled recep- tors (7-11). GIT1 is essential in cell proliferation and migration, and is located in the cytoplasmic membrane of focal adhesions and composite structures, such as inclusion 
bodies of cells in vivo (8-11). Yin et al (26) indicated that the phosphorylation of GIT1 was enhanced in the presence of Src by angiotensin II and EGF. In the present study, GIT1 was observed to be critical for chondrocyte proliferation, migration and apoptosis via Src regulation induced by PDGF. Furthermore, Menon et al (27) found that GIT1 is a key regulator of receptor activator of nuclear factor $-\kappa \mathrm{B}$ signaling, comparable with Src-mediated tyrosine phosphorylation and phosphorylation of PLC $\gamma 1$, and regulates osteoclast function and bone mass. However, recent studies have focused on the effects of GIT1 on cell migration in osteoblasts rather than chondrocytes. For example, Ren et al (15) demonstrated that GIT1 tyrosine 321 phosphorylation is required for association with FAK, FAK activation in focal adhesions and for PDGF-induced osteoblastic cell migration. In the present study, downregulation of GIT1 was identified to inhibit the effects of PDGF on promotion of chondrocyte proliferation and migration, and inhibition of apoptosis in cartilage cells, indicating that GIT1 plays a similar role to PDGF in the promotion of chondrocyte proliferation and migration, and inhibition of apoptosis.

In conclusion, PDGF may promote chondrocyte proliferation and migration, and inhibit apoptotic cell death by upregulation of GIT1 expression and Src phosphorylation. However, the underlying mechanisms of bone healing are complex and intertwined and the results of the present study indicated that multiple signaling molecules are involved. Thus, further investigation is required into the role of PDGF in other signaling pathways.

\section{References}

1. Yang D, Chen J, Jing Z and Jin D: Platelet-derived growth factor (PDGF)-AA: A self-imposed cytokine in the proliferation of human fetal osteoblasts. Cytokine 12: 1271-1274, 2000.

2. Filardo G, Kon E, Di Martino A, Iacono F and Marcacci M: Arthroscopic second-generation autologous chondrocyte implantation: A prospective 7-year follow-up study. Am J Sports Med 39: 2153-2160, 2011.

3. Brandl A, Angele P, Roll C, Prantl L, Kujat R and Kinner B: Influence of the growth factors PDGF-BB, TGF-betal and bFGF on the replicative aging of human articular chondrocytes during in vitro expansion. J Orthop Res 28: 354-360, 2010.

4. Nash TJ, Howlett CR, Martin C, Steele J, Johnson KA and Hicklin DJ: Effect of platelet-derived growth factor on tibial orteotomies in rabbits. Bone 15: 203-208, 1994.

5. Lee JY, Nam SH, Im SY, Park YJ, Lee YM, Seol YJ, Chung CP and Lee SJ: Enhanced bone formation by controlled growth factor delivery from chitosan-based biomaterials. J Control Release 78: 187-197, 2002.

6. Schmidt MB, Chen EH and Lynch SE: A review of the effects of insulin-like growth factor and platelet derived growth factor on in vivo cartilage healing and repair. Osteoarthritis Cartilage 14: 403-412, 2006

7. Manabe R, Kovalenko M, Webb DJ and Horwitz AR: GIT1 function sin a motile, multi-molecular signaling complex that regulates protrusive activity and cell migration. J Cell Sci 115 1497-1510, 2002.
8. Premont RT, Claing A, Vitale N, Freeman JL, Pitcher JA, Patton WA, Moss J, Vaughan $M$ and Lefkowitz RJ: Beta2-Adrenergic receptor regulation by GIT1, a GIT1, a G protein coupled receptor kinase-associated ADP ribosylation factor and GTPase-activating protein. Proc Natl Acad Sci USA 95: 14082-14087, 1998.

9. Rui Z, Li X, Fan J, Ren Y, Yuan Y, Hua Z, Zhang N and Yin G: GIT1Y321 phosphorylation is required for ERK1/2- and PDGF-dependent VEGF secretion from osteoblasts to promote angiogenesis and bone healing. Int J Mol Med 30: 819-825, 2012.

10. Schmalzigaug R, Phee H, Davidson CE, Weiss A and Premont RT: Differential expression of the ARF GAP genes GIT1 and GIT2 in mouse tissues. J Histochem Cytochem 55: 1039-1048, 2007.

11. Claing A, Perry SJ, Achiriloaie M, Walker JK, Albanesi JP, Lefkowitz RJ and Premont RT: Multiple endocytic pathways of $\mathrm{G}$ protein-coupled receptors delineated by GIT1 sensitivity. Proc Natl Acad Sci USA 97: 1119-1124, 2000.

12. Zhang H, Webb DJ, Asmussen H and Horwitz AF: Synapse formation is regulated by the signaling adaptor GITI. J Cell Biol 161: 131-142, 2003.

13. Chaturvedi LS, Marsh HM and Basson MD: Src and focal adhesion kinase mediate mechanical strain-induced proliferation and ERK1/2 phosphorylation in human $\mathrm{H} 441$ pulmonary epithelial cells. Am J Physiol Cell Physiol 292: C1701-C1713, 2007.

14. Shen E, Fan J, Chen R, Yee SP and Peng T: Phospholipase Cgamma1 signalling regulates lipopolysaccharide-induced cyclooxygenase-2 expression in cardiomyocytes. J Mol Cell Cardiol 43: 308-318, 2007.

15. Ren Y, Yu L, Fan J, Rui Z, Hua Z, Zhang Z, Zhang N and Yin G: Phosphorylation of GIT1 tyrosine 321 is required for association with FAK at focal adhesions and for PDGF-activated migration of osteoblasts. Mol Cell Biochem 365: 109-118, 2012.

16. Golden LH and Insogna KL: The expanding role of PI3-kinase in bone. Bone 34: 3-12, 2004.

17. Fredriksson L, Li H and Eriksson U: The PDGF family: Four gene products form five dimeric isoforms. Cytokine Growth Factor Rev 15: 197-204, 2004.

18. Claesson-Welesh L: Platelet-derived growth factor receptor signals. J Biol Chem 269: 32023-32026, 1994.

19. Livak KJ and Schmittgen TD: Analysis of relative gene expression data using real-time quantitative PCR and the $2^{-\Delta \Delta C}$ method. Methods 25: 402-408, 2001.

20. Marcopoulou CE, Vavouraki HN, Dereka XE and Vrotsos IA: Proliferative effect of growth factors TGF-betal, PDGF-BB and rhBMP-2 on human gingival fibroblasts and periodontal ligament cells. J Int Acad Periodontol 5: 63-70, 2003.

21. Oreffo RO: Growth factors for skeletal reconstruction and fracture repair. Curr Opin Investig Drugs 5: 419-423, 2004.

22. Adams CS and Shapiro IM: The fate of the terminally differentiated chondrocyte: Evidence for microenvironmental regulation of chondrocyte apoptosis. Crit Rev Oral Biol Med 13: 465-473, 2002.

23. Chaturvedi LS, Gayer CP, Marsh HM and Basson MD: Repetitive deformation activates Src-independent FAK-dependent ERK motogenic signals in human Caco-2 intestinal epithelial cells. Am J Physiol Cell Physiol 294: C1350-C1361, 2008.

24. Morita N, Iizuka K, Murakami T and Kawaguchi H: N-terminal kinase and c-Src are activated in human aortic smooth muscle cells by pressure stress. Mol Cell Biochem 262: 71-78, 2004.

25. Ren K, Ma Y, Huang Y, Liang W, Liu F, Wang Q, Cui W, Liu Z, Yin G and Fan W: Periodic mechanical stress activates MEK1/2-ERK1/2 mitogenic signals in rat chondrocytes through Src and PLC $\gamma 1$. Braz J Med Biol Res 44: 1231-1242, 2011.

26. Yin G, Zheng Q, Yan C and Berk BC: GIT1 is a scaffold for ERK1/2 activation in focal adhesions. J Biol Chem 280: 27705-27712, 2005.

27. Menon P, Yin G, Smolock EM, Zuscik MJ, Yan C and Berk BC: GPCR kinase 2 interacting protein 1 (GIT1) regulates osteoclast function and bone mass. J Cell Physiol 225: 777-785, 2010. 